SHS Web of Conferences 7, 01004 (2014)

DOI: $10.1051 /$ shsconf / 20140701004

C) Owned by the authors, published by EDP Sciences, 2014

\title{
The Decision of Enterprises during the Process of Technological Innovation Diffusion Based on Real Option Theory
}

\author{
Yang Guozhong \\ Business School, Central South University, 410083 Changsha Hunan, China
}

\begin{abstract}
Based on real option theory, the article analyses the decision-making of enterprises during the process of technological innovation diffusion under an uncertain circumstance. Under the assumption that the returns of enterprises follow geometric Brownian motion, the article firstly estimates the transition value of imitating technology innovators and the average latency of imitation, then it analyses the influence of every parameter on the diffusion process. It can be concluded that both the market demand and the rate have significant effects on the diffusion rate of innovative technology.
\end{abstract}

Keywords. technological innovation; technological innovation diffusion; real option; geometric brownian motion

\section{Introduction}

The process of technological innovation diffusion (TID) involves many dynamic and uncertain factors; the academic world has been slowly begun to focus on its uncertainty [1-2]. There are scholars who have studied the diffusion process of uncertainty, especially about the diffusion mechanism and diffusion model, and many valuable conclusions are obtained. However, TID is full of random characteristic, and the decision reflects the multiphase and delayed. Facing the innovation income, cost and the highly uncertain environment, the random process theory can well solve the decision problem in the process of TID. For example, Ma Lei [3] formulated a model of TID based on Markov chain. Tang Zhenpeng [4] thought the investment opportunity of enterprise innovation as a compound American call option held by the enterprise. In the existing literatures, the diffusion models for technological innovation under uncertainty environment need for further research. First of all, most of existing models are deterministic models [5-6], but it has obviously difference between the theory process to describe diffusion model and the actual diffusion process because of the behavior of TID process, access to information and random factors. Though deterministic model can describe the overall trend diffusion, it cannot describe the decision behavior and the stochastic characteristics of adoption time. Secondly, though the model is studied under the complex and uncertainty environment, it is only on the macro level. However, the macro model cannot meet the actual because the key is the people involved in the decision-making. Finally, there are some individual decision-making models, but the process of diffusion of technology innovation uncertainty is not considered as a whole. In view of this, beginning from the individual decision facing uncertainty, this article puts forward the similarity coefficient of enterprise technology innovation and imitation and formulates the decisionmaking model based on innovation revenue and cost and the similarity of enterprise technology. 


\section{Assumptions of model}

We assume that a new technology is put into the market and its innovator chooses to maintain the technology monopoly, the market return of the new product is $R(t, 1)$ at the time $t$, it changes by following the geometric Brown motion:

$$
d R(t, 1) / R(t, 1)=u d t+\sigma d w
$$

Where, $\mu_{\text {denotes the expected growth rate for the corresponding return and }} \mu>0, \sigma$ is volatility and $\sigma>0, d w$ is a standard Wiener process increment.

We assume that the earning of any one potential adopter is a fixed constant A. After potential adopters have imitated innovation technology, their technologies and innovation technology are more and more similar, their returns are close to $R(t, 1)$. We further assume, at the time $t$, technology similarity is $p(0<p<1)$, and $R(t, 1)$ meets the following formula:

$$
R(t, p)=p R(t, 1)
$$

\section{Model formulation}

\subsection{The option value}

According to above assumptions, at the time ${ }^{t}$, enterprise value equates that fixed income $A$ adds present value of income stream and minuses the present value of cost, i.e.:

$$
\begin{aligned}
& \pi(t, p)=\max _{T}\left\{E _ { t } \left[\int_{t}^{T} A e^{-r(\tau-t)} d \tau\right.\right. \\
& \left.\left.+\int_{T}^{\infty} R(\tau, p) e^{-r(\tau-t)} d \tau-p I e^{-r(T-t)}\right]\right\}
\end{aligned}
$$

Where $T$ denotes the time of occurrence for imitation. The equation can be given by

$$
\pi(t, p)=\frac{A}{r}+F[R(t, 1), p]
$$

Where $F[R(t, 1), p]$ is a conversion option, the value is:

$$
\begin{aligned}
& F[R(t, 1), p]=\max _{T}\left\{E _ { t } \left\{\int_{T}^{\infty}[R(\tau, p)-A] e^{-r(\tau-t)} d \tau\right.\right. \\
& \left.\left.-p I e^{-r(T-t)}\right\}\right\}
\end{aligned}
$$

If $V[R(T, 1), p]$ refers to intrinsic value which conversion occurs, then

$$
V[R(T, 1), p]=E_{T}\left\{\int_{T}^{\infty}[R(\tau, p)-A] e^{-r(\tau-T)} d \tau-p I\right\}
$$

Let $\tau-T=S$, according to Fubini Theory ${ }^{[7]},(6)$ will be become to

$$
V[R(T, 1), p]=\frac{p R(T, 1)}{r-u}-\frac{A}{r}-p I
$$

\subsection{The threshold of imitation}

If time is continuous, $F$ also follows by the random process:

$$
d F=\left(\frac{\partial F}{\partial R} R u+\frac{\partial F}{\partial t}+\frac{1}{2} \frac{\partial^{2} F}{\partial R^{2}} R^{2} \sigma^{2}\right) d t+\frac{\partial F}{\partial R} \sigma R d w
$$

The expected revenue of options during the unit time is

$$
E(d F)=\left(\frac{\partial F}{\partial R} R u+\frac{\partial F}{\partial t}+\frac{1}{2} \frac{\partial^{2} F}{\partial R^{2}} R^{2} \sigma^{2}\right) d t
$$


By the assumption of no arbitrage equilibrium of real options, the expected rate of return should be equal to the average income, namely, the partial differential equation of option value is described as the following:

$$
\frac{\partial F}{\partial R} R u+\frac{\partial F}{\partial t}+\frac{1}{2} \frac{\partial^{2} F}{\partial R^{2}} R^{2} \sigma^{2}=r F
$$

We assume further that cash time is fixed. Therefore, $\frac{\partial F}{\partial t}=0$ and (10) is simplified into

$$
\frac{1}{2} \frac{\partial^{2} F}{\partial R^{2}} R^{2} \sigma^{2}+\frac{\partial F}{\partial R} R u-r F=0
$$

Its general solution is $B R^{\beta_{1}}+C R^{\beta_{2}}$, where, $\beta_{1}$ and $\beta_{2}$ are the two solutions of characteristic equation (12):

$$
\frac{1}{2} \sigma^{2} \beta^{2}-\left(\frac{1}{2} \sigma^{2}-u\right) \beta-r=0
$$

They are

$$
\begin{aligned}
& \beta_{1}=\frac{1}{2}-\frac{u}{\sigma^{2}}+\sqrt{\left(\frac{u}{\sigma^{2}}-\frac{1}{2}\right)^{2}+\frac{2 r}{\sigma^{2}}} \\
& \beta_{2}=\frac{1}{2}-\frac{u}{\sigma^{2}}-\sqrt{\left(\frac{u}{\sigma^{2}}-\frac{1}{2}\right)^{2}+\frac{2 r}{\sigma^{2}}}
\end{aligned}
$$

In order to ensure the option can be executed, we assume $r>u$, therefore, $\beta_{1}>1$ and $\beta_{2}<0$. From the initial conditions $F(0)=0$, we know that $\beta_{2}<0$ has no significance. So $F(R)=B R^{\beta_{1}}$, and $F$ and $V$ must meet with value matching conditions $F[R(T, 1)]=V[R(T, 1)]$ and the smooth pasting condition $F_{R}[R(T, 1)]=V_{R}[R(T, 1)]$, namely

$$
\left\{\begin{array}{l}
B R(T, 1)^{\beta_{1}}=\frac{p R(T, 1)}{r-u}-\frac{A}{r}-p I \\
B \beta_{1} R(T, 1)^{\beta_{1}-1}=\frac{p}{r-u}
\end{array}\right.
$$

We solve this equation and the solutions are

$$
\begin{aligned}
& R(T, 1)=\frac{(r-u) \beta_{1}(A+r p I)}{\left(\beta_{1}-1\right) r p} \\
& B=\frac{p}{\beta_{1}(r-u)} R(T, 1)^{\left(1-\beta_{1}\right)}
\end{aligned}
$$

(13) is an imitation decision model for potential adopters, where $R(T, 1)$ is the threshold of imitation. Adopters will imitate imitation innovation technology when the benefits exceed $R(T, 1)$, or else, they wait for imitation. At this time, the value of conversion option is

$$
F[R(t, 1), p]= \begin{cases}\frac{(A+r I p)}{\left(\beta_{1}-1\right) r}\left[\frac{R(t, 1)}{R(T, 1)}\right]^{\beta_{1}}, & R(t, 1)<R(T, 1) \\ \frac{p R(t, 1)}{r-u}-\frac{A}{r}-p I, & R(t, 1) \geq R(T, 1)\end{cases}
$$

According to (15) and (4), at the time $t$, the instantaneous value of the potential adopters is

$$
\pi(t, p)=\frac{(A+r I p)}{\left(\beta_{1}-1\right) r}+\frac{A}{r}
$$

\subsection{Accessibility to the imitation threshold}


If $x$ follows the arithmetic Brown motion $d x=\alpha d t+\sigma d w, x$ from the initial value $x_{0}$ to the threshold $a$ for the first arrival time, the probability density function is given by

$$
g\left(t ; x_{0}, a\right)=\frac{\left|a-x_{0}\right|}{\sigma \sqrt{2 \pi t^{3}}} \exp \left[-\frac{\left(a-x_{0}-\alpha t\right)^{2}}{2 \sigma^{2} t}\right]
$$

Let $x=\ln R(t, 1)$, then $d x=\left(u-\sigma^{2} / 2\right) d t+\sigma d w$ follows the geometric Brown motion. Assuming the initial return for the innovator is $R_{0}\left(R_{0}<R(T, 1)\right)$, let $t^{*}=\inf \left\{t \geq 0: R(t, 1)=R(T, 1), R(0,1)=R_{0}\right\}$, according to (17), the density function at the first arrival time is

$$
\begin{aligned}
& f\left[t ; R_{0}, R(T, 1)\right] \\
& =\frac{\ln \left[\frac{R(T, 1)}{R_{0}}\right]}{\sigma \sqrt{2 \pi t^{3}}} \exp \left\{-\frac{\left[\ln \left(\frac{R(T, 1)}{R_{0}}\right)-\left(u-\frac{1}{2} \sigma^{2}\right) t\right]^{2}}{2 \sigma^{2} t}\right\}
\end{aligned}
$$

When $u-\frac{1}{2} \sigma^{2}>0, \quad R$ can reach to $R(T, 1)$ in the limited time and $E\left(t^{*}\right)=\frac{\ln \left[R(T, 1) / R_{0}\right]}{\left(u-\sigma^{2} / 2\right)}, \quad \operatorname{var}\left(t^{*}\right)=\frac{\sigma^{2} \ln \left[R(T, 1) / R_{0}\right]}{\left(u-\sigma^{2} / 2\right)^{3}} ; \quad$ when $u-\frac{1}{2} \sigma^{2}<0 \quad, \quad R$ maybe reach $R(T, 1)$, which the arrival probability is $\left[\frac{R(T, 1)}{R_{0}}\right]^{\frac{2 u}{\sigma^{2}}-1}$, but expectation and variance of waiting time for arriving imitation threshold are infinite. How long will it take to reach the threshold of imitation, is the rate of technological diffusion problem. Thus,

$$
\partial E\left(t^{*}\right) / \partial p=\frac{\partial E\left(t^{*}\right)}{\partial R(T, 1)} \cdot \frac{\partial R(T, 1)}{\partial p}<0
$$

\section{Model analysis}

\subsection{The analysis of key parameters}

We will analyze firstly about the derivatives of each variable by $\sigma, u, r, A 、 I, p$. When the analytical method cannot be determined, we will analyze it by the numerical simulation method.

According to (13), the following equation will be gotten:

$$
\begin{aligned}
& \ln R(T, 1)=\ln (r-u)+\ln \beta_{1}+\ln (A+r p I) \\
& -\ln \left(\beta_{1}-1\right)-\ln r-\ln p
\end{aligned}
$$

After deriving various parameters, the results will be

$$
\begin{aligned}
& \frac{\partial \ln R(T, 1)}{\partial \sigma}=-\frac{\frac{\partial \beta_{1}}{\partial \sigma}}{\left(\beta_{1}-1\right) \beta_{1}}>0 \\
& \frac{\partial \ln R(T, 1)}{\partial A}=\frac{1}{A+I p r}>0 \\
& \frac{\partial \ln R(T, 1)}{\partial I}=\frac{r p}{A+I r}>0 \\
& \frac{\partial \ln R(T, 1)}{\partial p}=-\frac{A}{p(A+r p I)}<0
\end{aligned}
$$


For it is very difficult to judge $\partial \ln R(T, 1) / \partial u$ and $\partial \ln R(T, 1) / \partial r$ are positive or negative by analytical method, we judge how $u$ and $r$ will affect $R(T, 1)$ by the method of numerical simulation. Let $r=0.1 、 \sigma=0.2 、 A=1 、 I=5 、 p=0.5$, and we ensure $u-\frac{1}{2} \sigma^{2}>0$ and $r>u$. The effect of $R(T, 1)$ affected by $u$ is shown in Figure 1. Obviously, $\partial R(T, 1) / \partial u<0$.

Similarly, le t $u=0.05 、 \sigma=0.2 、 A=1 、 I=5 、 p=0.5$, The effect of $R(T, 1)$ affected by $r$ is shown in Figure 2. Obviously, $\partial R(T, 1) / \partial r>0$.

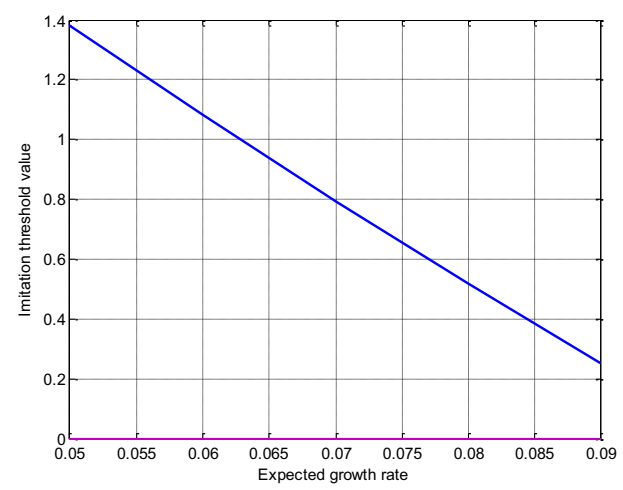

Figure 1. The expected growth rate and the threshold value

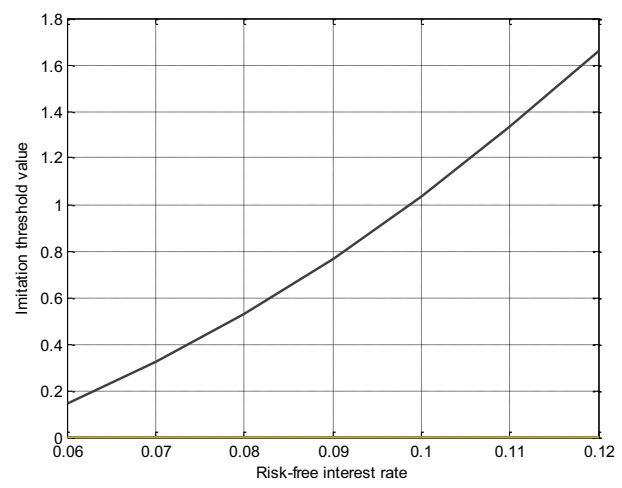

Figure 2. Risk-free interest rate and the threshold value

Analysis with similar analytical, $\frac{\partial \pi(t, p)}{\partial \sigma}, \frac{\partial \pi(t, p)}{\partial u}, \frac{\partial \pi(t, p)}{\partial A}, \frac{\partial \pi(t, p)}{\partial I}, \frac{\partial \pi(t, p)}{\partial p}$ are positive. For it is difficult to analyze the effect of $\pi(t, p)$ affected by analytical method, we judge it by the method of numerical simulation. Let $u=0.05 、 \sigma=0.2 、 A=1 、 I=5 、 p=0.5$, and we ensure $r>u$. The result is shown in Figure 3. Obviously, $\partial \pi(t, p) / \partial r<0$. 


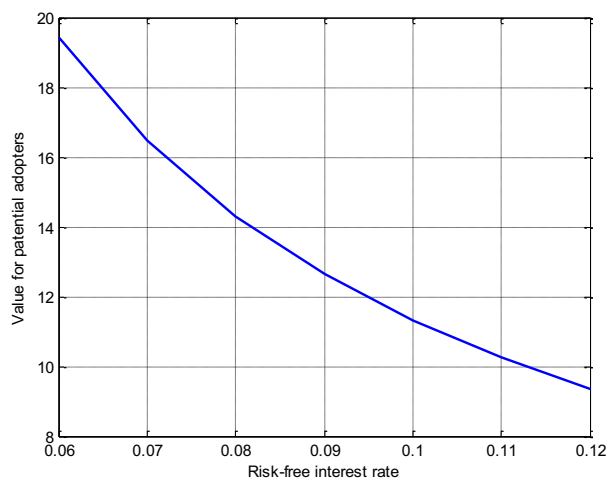

Figure 3. The risk-free rate and value of potential adopters

Thirdly, we analyze $E\left(t^{*}\right)$.

For $\partial E\left(t^{*}\right) / \partial R(T, 1)>0$, the influence of each parameter on $E\left(t^{*}\right)$ and on $R(T, 1)$ are same, i.e.

$$
\frac{\partial E\left(t^{*}\right)}{\partial \sigma}>0, \frac{\partial E\left(t^{*}\right)}{\partial u}<0, \frac{\partial E\left(t^{*}\right)}{\partial A}>0, \frac{\partial E\left(t^{*}\right)}{\partial I}>0, \frac{\partial E\left(t^{*}\right)}{\partial p}<0, \frac{\partial E\left(t^{*}\right)}{\partial r}>0
$$

\subsection{The analysis results and economic meaning}

All of analysis results are summarized as Table 1.

Table 1. All of analysis results.

\begin{tabular}{|c|c|c|c|c|c|c|}
\hline & $\sigma$ & $\mathrm{u}$ & $\mathrm{A}$ & $\mathrm{r}$ & $\mathrm{I}$ & $\mathrm{p}$ \\
\hline $\mathrm{R}(\mathrm{T}, 1)$ & + & - & + & + & + & - \\
\hline$\Pi(\mathrm{t}, \mathrm{p})$ & + & + & + & - & + & + \\
\hline $\mathrm{E}\left(\mathrm{t}^{*}\right)$ & + & - & + & + & + & - \\
\hline
\end{tabular}

Notes: "+" indicates the variable increases as the parameter increases, "-" indicates the variable decreases as the parameters increases

The uncertainty of earnings by imitating innovative technology is larger, imitation threshold, the enterprise value and the average waiting time are added; with increasing of uncertainty, option is delayed. Expected earnings growth rate makes the imitation threshold reduce, and the transformation of the average waiting time is reduced. Increase of risk-free interest rate makes the investment threshold and average waiting time of investment increase. The threshold value, enterprise value and the average waiting time will increase if revenue and conversion costs of enterprises without adopting innovation technology. With the degree of similarity being bigger, the development threshold and the average waiting time will be reduced. When the imitation technology is closer with innovation technology, more enterprises are willing to imitate them, therefore, technology innovation diffusion is faster and the enterprise value is greater.

\section{Conclusion}

This paper analyzes the micro level behavior of enterprises in the process of technology innovation diffusion based on the real option theory. According to these analyses, the increase of diffusion rate 
will reduce the uncertainty of technology innovation. Whether the new technology is welcomed by the market, enterprises will choose firstly an existing technology and not to imitate the new technology. As the expected increase in growth rate will make the technological innovation diffusion speed quickly, enterprises have to follow a leader one in technology, so as to accelerate the popularization of innovation technology and promote the innovator to begin with a new round of innovation. Increase of market interest rates will make speed of technology diffusion become slower, and no enterprise is willing to invest because the present value of future cash flow is reduced. In short, for any enterprise, it can increase its income until it produce and sell lots of products which consumers are satisfied with. In order to meet it, leading enterprise continue to innovate to maintain the leading position while other enterprises imitate innovation technology.

\section{Acknowledgement}

Supported by Social Science Foundation of Hunan Province (GN:13YBA355)

\section{References}

1. Doraszelski U. Innovations, Improvements, and the Optimal Adoption of New Technologies[J]. Journal of Economic Dynamics and Control, 2002, 28(7):1461-1480.

2. Huisman K J M, Kort P M. Strategic Technology Investment Under Uncertainty[J]. OR Spectrum, 2002, 24(1):79-98.

3. Ma Lei, Luo Jianqiang, Huang Keji, Ye Rui. Analysis of Technical Innovation Diffusion Theory and its Simulation Based on Markov Chain [J]. Science of Science and Management of S. \& T. 2012, 33(2):44-48.

4. Tang Zhenpeng, Liu Guoxin. Decision-making of an Imitation Innovation Using a Real Option Approach [J].Journal of WUT (Information \& Management Engineering). 2002, 24(6):17-19.

5. Reimer S. Getting by in Time and Space Fragmented Work in Local Authorities [J]. Economic Geography, 2000, 75(2): 475-491

6. Zhao Weishuang, Liu Tao. Analysis of Technology Innovation Diffusion Environment and Influence to Diffusion model [J]. On Economic Problems, 2008(12): 63-65.

7. Yan Shijian, Wang Junxiang, Liu Xiufang. Base of Probability[M]. Beijing: Science Press, 1982 